\title{
A CLOUD-BASED PLATFORM SUPPORTING GEOSPATIAL COLLABORATION FOR GIS EDUCATION
}

\author{
Xiaoqiang Cheng ${ }^{\mathrm{a}}$, Zhipeng Gui ${ }^{\mathrm{b}}$, Kai Hu${ }^{\mathrm{a}}$, Shuang Gao ${ }^{\mathrm{a}}$, Ping Shen ${ }^{\mathrm{a}}$, Huayi $\mathrm{Wu}^{\mathrm{a}}$ \\ ${ }^{a}$ LIESMARS, Wuhan University, China - (carto, hukai, shuanggao, shenping, wuhuayi)@whu.edu.cn \\ ${ }^{\mathrm{b}}$ School of Remote Sensing and Information Engineering, Wuhan University, China - zhipeng.gui@ whu.edu.cn
}

Commission VI, WG 1/3

KEY WORDS: cloud computing, geospatial collaboration, model sharing, GIS education,

\begin{abstract}
:
GIS-related education needs support of geo-data and geospatial software. Although there are large amount of geographic information resources distributed on the web, the discovery, process and integration of these resources are still unsolved. Researchers and teachers always searched geo-data by common search engines but results were not satisfied. They also spent much money and energy on purchase and maintenance of various kinds of geospatial software. Aimed at these problems, a cloud-based geospatial collaboration platform called GeoSquare was designed and implemented. The platform serves as a geoportal encouraging geospatial data, information, and knowledge sharing through highly interactive and expressive graphic interfaces. Researchers and teachers can solve their problems effectively in this one-stop solution. Functions, specific design and implementation details are presented in this paper. Site of GeoSquare is: http://geosquare.tianditu.com/
\end{abstract}

\section{INTRODUCTION}

With the advancement of sensors and information technologies, there are more and more geographical information resources (GIRs), including data, algorithms, application and models, hosted on the web for research, teaching and learning(Gong and $\mathrm{Wu}, 2012)$. However, due to the irregular distribution and poor organization of GIRs, it is too difficult for teachers to find appropriate geographic data and processing tools in their teaching(Ivánová et al., 2013). Although GI data and software can be purchased, they cost much money and their setup, maintenance and update are not a trivial. Even now, it is still a challenging task to discover and utilize demanded online GIRs for researchers and teachers(Smits and Friis-Christensen, 2007). How to help teachers to solve their geospatial educational troubles effectively and efficiently becomes an urgent problem. In this context, we designed a geospatial collaboration framework called GeoSquare, which implemented on the basis of widely used private cloud solution: Cloud Stack. Through integrating GIRs discovery, processing and orchestration in one web portal, GeoSquare can help researchers and teachers searching and utilizing online resources at a low cost in an efficient and harmonious way.

The remainder of this paper is organized as follows: Section 2 presents the design and implementation of GeoSquare; Section 3 describes GeoSquare's main function; Section 4 introduces the application of GeoSquare briefly.

\section{DESIGN \& IMPLEMENTATION}

GeoSquare was designed in a three-tier architecture including Resource tier, Server tier and Application tier, among which Server tier implements core functions, Resource tier manages database and Application tier is responsible for user interface and system maintenance. Server tier consists of workflow engine capable of geographic modelling, image processor in charge of interpreting remote sensing image, WMS crawler for harvesting web map services and WMS monitor used to obtain status information of web map service etc. Resource tier manage a database which stores geographic data, service, geoprocessing model and their metadata. These GIRs are collected from the web and updated on schedule. Web portal in the application tier is an interactive user interface allowing people access its function crafted by GeoChianing and data registered in registry center. Teachers can search geospatial data on-demand and use GeoChaining to integrate simple geoprocessing tools into a sophisticated model which should be shared in the process of teaching. Of course, Students also will share their models and collaborate with each other through GeoSquare.

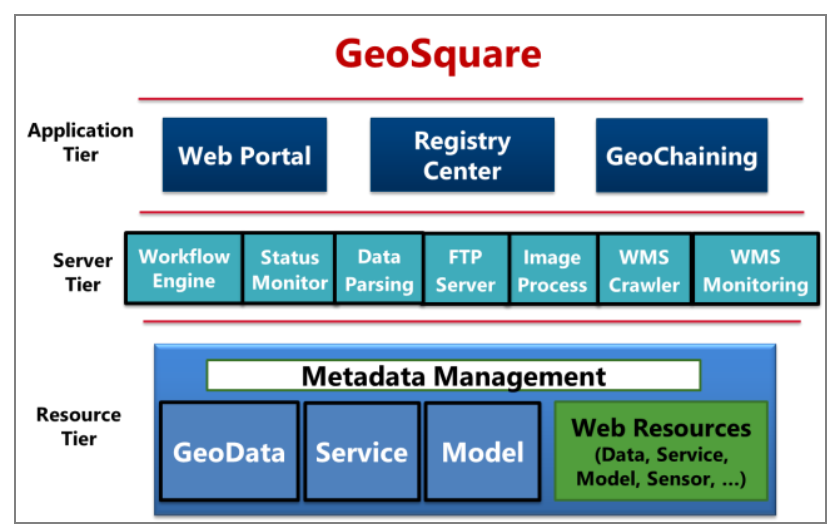

Figure 1 Architecture of GeoSquare

In terms of technical aspects, GeoSquare was implemented based on web technologies such as the enterprise Web portal, Rich Internet Applications, Java User Interface toolkits, and workflow engines. Most of these technologies are built upon open source standards, which ensure the delivery of contentrich and cross-platform applications.

To extend the scalability and usability of the GeoSquare, we promoted the architectures of the infrastructure by Cloud Stack, 


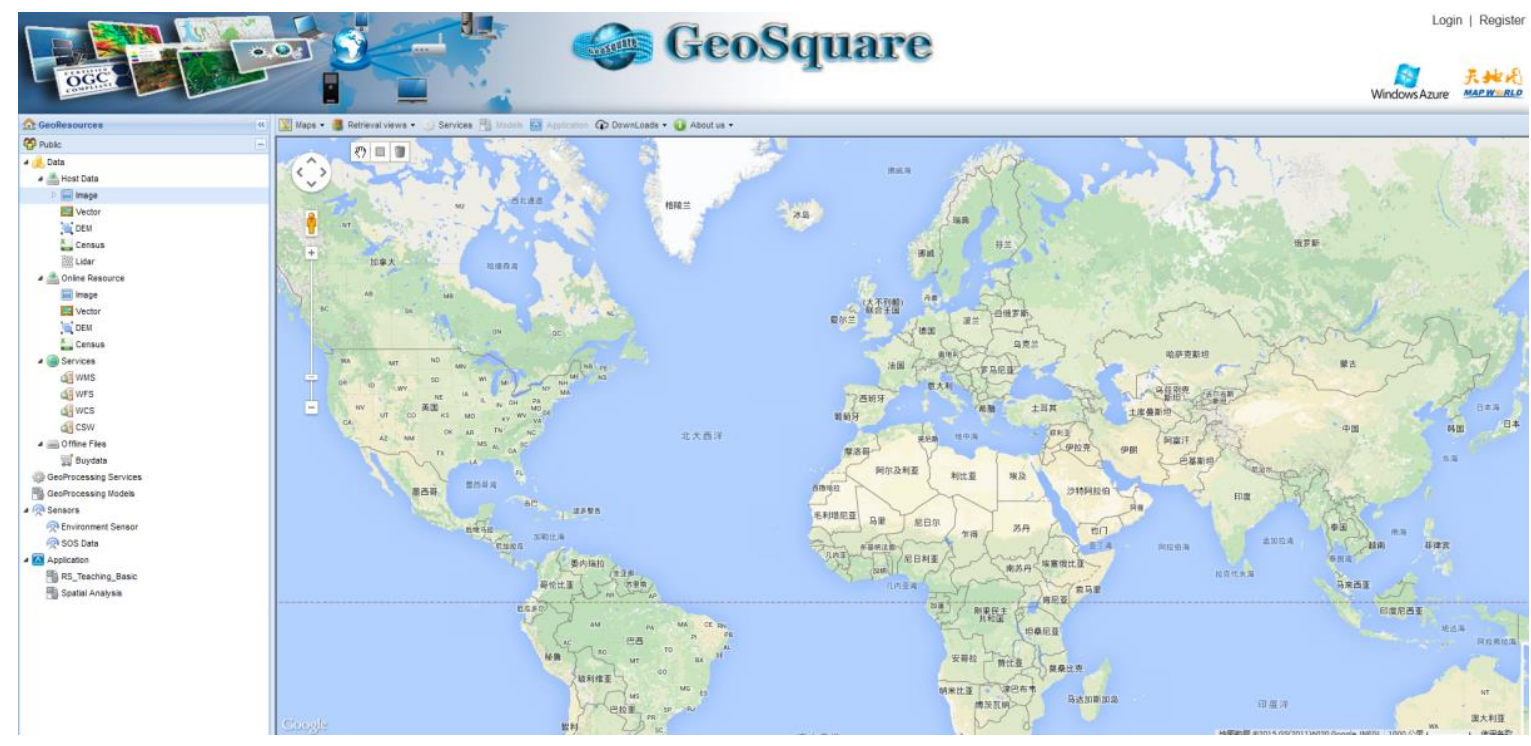

Figure 2 Interface of GeoSquare

through introducing one-data-node-multiple-application-node architecture, which is convenient for scale up by setting the application-nodes' numbers. The MySQL-cluster is also used to promote the reliability of the metadata databases. The services workflow engines are expended in the load balance mechanisms in certain scales of server clusters. All of the MySQL-cluster nodes, the GeoSquare's application nodes and the service workflow engines are made into templates of the Kernel-based Virtual Machine (KVM) which provided by the Cloud Stack. By using control center of the web interfaces of the Cloud Stack, the clusters' scale of applications, the databases, and the service workflow engines can be deliberately set under different internet environment.

\section{FUNCTIONS}

GeoSquare consists of server-side database and client-side user interface, which includes Tree view containing resources catalogue and Map view representing geographic data, as displayed in Figure 2. In this section, resources and operations supported in GeoSquare will be described respectively.

\subsection{Resources in GeoSquare}

All kinds of resources are registered in registration centre, which serves as a data manager of GeoSquare. Registration centre is designed to find and share Geographic data and Geographic Information Services, supported by a complete set of the database in the background, which maximize the sharing and interoperability of services. On the basis of the traditional registry, we evaluate the service quality and attach the quality score to our registered services(Wu et al., 2011), which could greatly improve the efficiency of service discovery in the query feedback. Currently, resources in registration center could be categorized into four groups, which are data, geoprocessing service, sensor observation services, and application. Each group is described as follow.

\subsubsection{Data}

Data are organized according to the data access mode, and the data is divided into host data, online data, Web Services data, and offline data four categories.

(1) Host data and online data consist of four data types: Image, Vector, Digital Elevation Model (DEM) and Census.
- Image includes numerous satellite images that cover most parts of China, North America and South America.

- Vector includes China's railway, highway and the coastline data etc. which is provided by the OpenStreetMap data. And the data is stored in Shapefile format.

- DEM contains the digital elevation model of some provinces in China.

- Census includes statistical data in multiple formats. Statistical data is empty now.

(2) Web Services data is obtained through the data service standard established by OGC, and the web data services contain WMS, WFS, WCS and CSW. And WFS and WCS are currently not available.

(3) Offline data can be obtained by contacting the data provider, our registration center only provide metadata of the pay data.

\subsubsection{Geoprocessing service}

Geoprocessing service has two subcategories that are atomic service and service chains. Atomic service is a single processing service that provides basic geospatial processing function. For example image geometric correction, buffer of vector, overlay of feature classes. Service chain, which is composed by a series of atomic services according to a geoprocessing model, is designed for solving the complicated geographical issues.

\subsubsection{Sensor observation service}

The Sensor Observation Service (SOS) is a web service to query real-time sensor data and sensor data time series and is part of the Sensor Web. The offered sensor data comprises descriptions of sensors themselves, which are encoded in the Sensor Model Language (SensorML), and the measured values in the Observations and Measurements (O\&M) encoding format.

The registration center has registered many sensor observation services provided by LIESMARS, and the sensor service is used to acquire soil humidity, air pressure, and wind speed observations etc. 


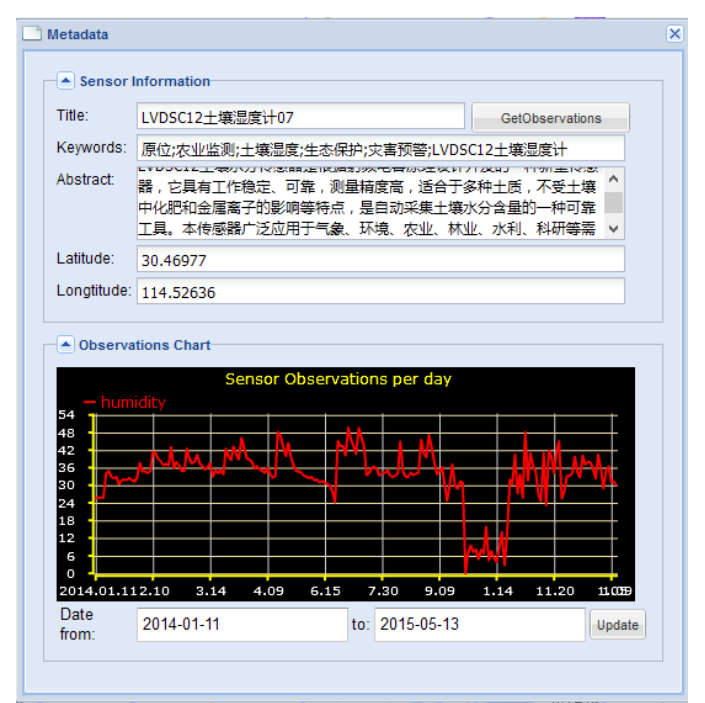

Figure 3 Live Data of Humidity Sensor

\subsubsection{Application}

Applications are out of the box tools embedded in GeoSquare, users can finish some lightweight work directly by using these tools online. Two applications are provided currently: lightweight remote sensing education platform and quadrat analysis tools.

(1) Remote sensing education platform utilizes services and data in the registration to process and analyse remote sensing image online. User interface developed by Flash is design for inputting parameters and displaying result. The platform has the four processing functions:

- Rectify: Geometric correction refers to the process of removing or correcting geometric error of remote sensing images.

- Classify: Supervised classification utilizes maximum likelihood classification, and the training samples are collected on the image by users.

- Assessment: This process use the GPS control points collected on the ground to verify correctness of the Classification.

- PostProcess: If the classification accuracy of a class is less than the required accuracy, and then makes adjustments to its region of interest.

(2) Quadrat analysis is designed to measure the point distribution patterns. The basic idea is to explore spatial distribution pattern of points through point density changes among grids. We set a random pattern as the standard distribution, compare the point density distribution with the standard distribution, and then decide the distribution to which the point pattern belongs.

\subsection{Operations in GeoSquare}

\subsubsection{Data retrieval}

GeoSquare provides data retrieval in the way of combining keywords and location of the resource which help users to find the resources they need efficiently. Depending on the scope to be retrieved, we divide the retrieval into Comprehensive Search and Categorized Search. Comprehensive Search will retrieve all types of resources in the Registry within the user's input extent. And Category Search retrieves the required resources within a specified category based on user's search condition. Users could also check the retrieval results in snapshot view (Figure 5).
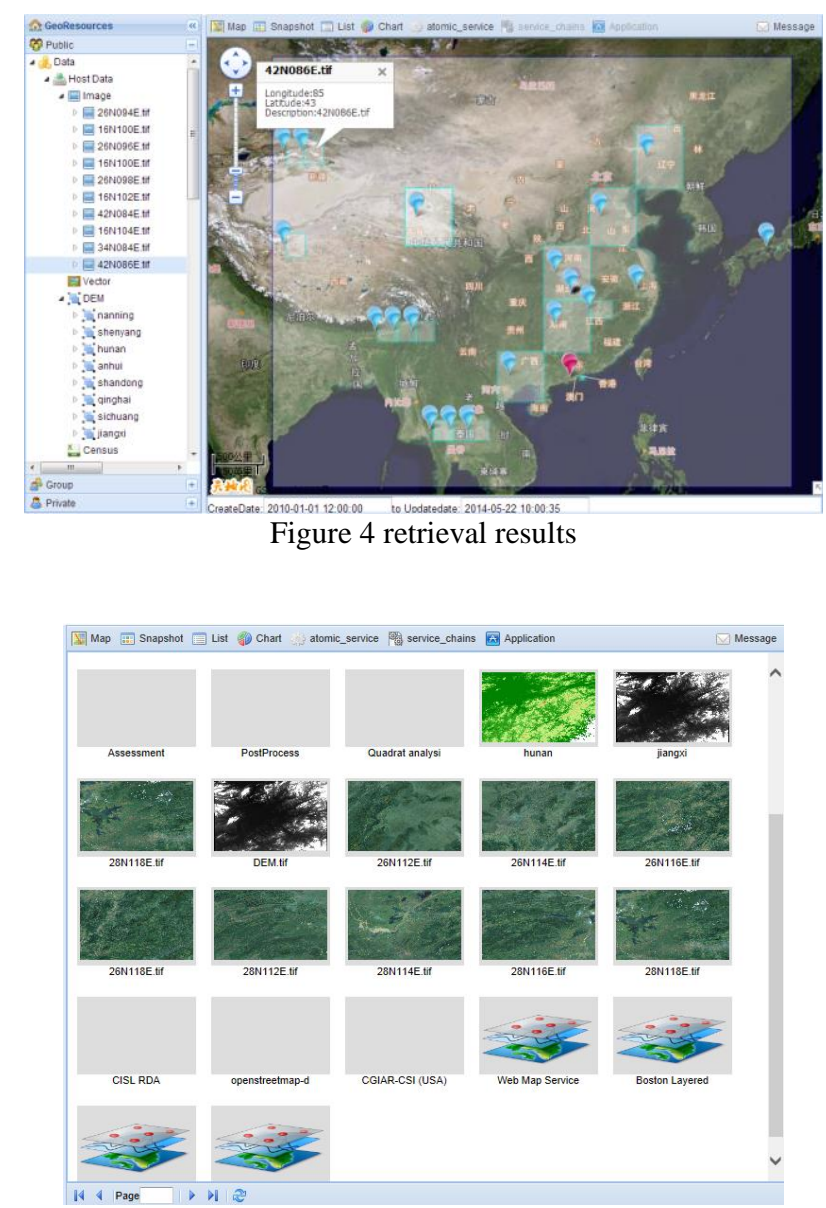

Figure 5 Snapshot of results

\subsubsection{Geoprocessing service invoke}

Currently, a large number of synchronous and asynchronous geoprocessing services have registered in the Registration Center. Before invoke of a geoprocessing service, users must find the right service conforming to their requirements. Using operations in GeoSquare as an example, users could find the "Atomic Service" node in the tree view, right-click on it, and then select "Query" in its context menu. Expand the newly created OpenRsService node, and then right click on the OpenRS_Statistic node and select "Invocate". The map view switches to atomic service execution view, and then fills in the following arguments. Finally click on the "invoke" button to start the atomic service, as shown in figure 6.

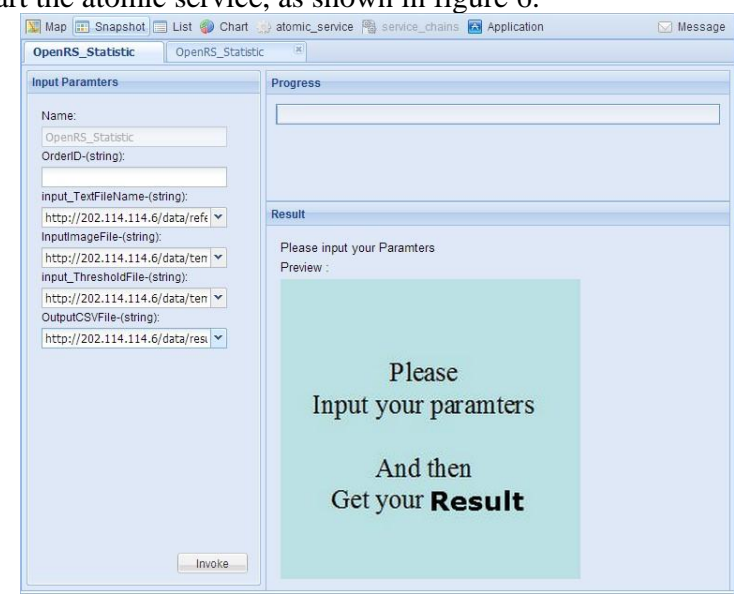

Figure 6 Interface of atom geoprocessing service 


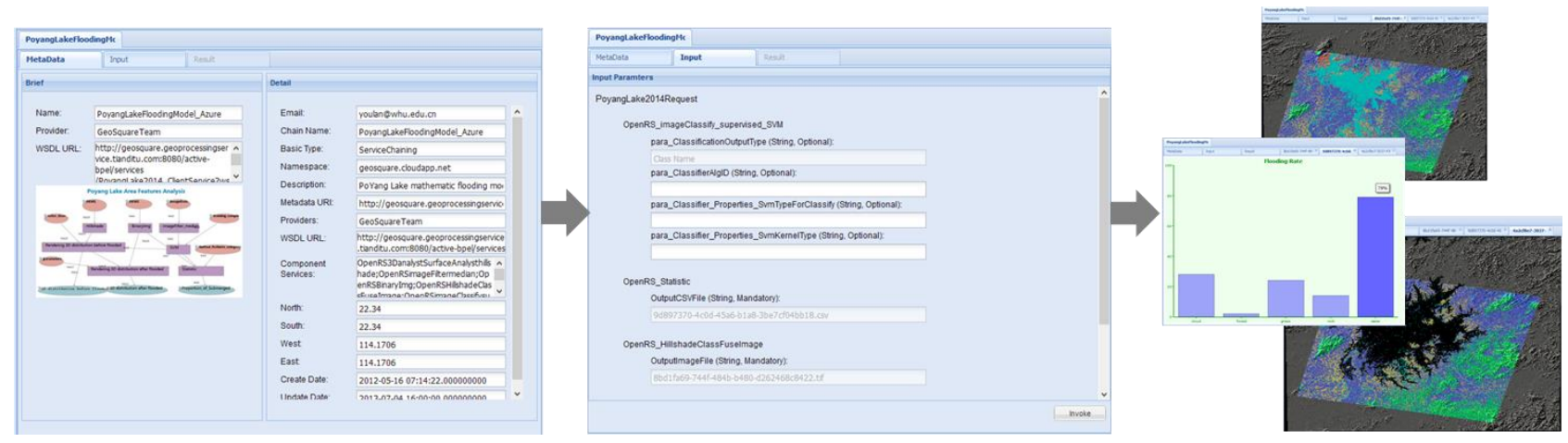

Figure 7 Full execution process of geoprocessing chain

In some cases, we might face a complex geographic information-related task, and we often need services chain which is a combination of multiple atomic Services. PoyangLakeFloodingModel is used to count the areas affected by the flooding during the Poyang lake flooding situation, which provide the basis for assessing the damage caused by floods. Here we will take the "PoyangLakeFloodingModel" as an example to display the process of querying and invoking the services chain, as shown in figure 7.

\subsubsection{Using Application}

Applications in GeoSquare are dependent tools which are developed by third-party. Users can either directly use data in registration center or upload their own data. Through simple interaction, such as drag, select or input the parameter, users will get results which can be display immediately or downloaded at will. Application's operations are usually easier than desktop softwares, and are suitable for teaching.

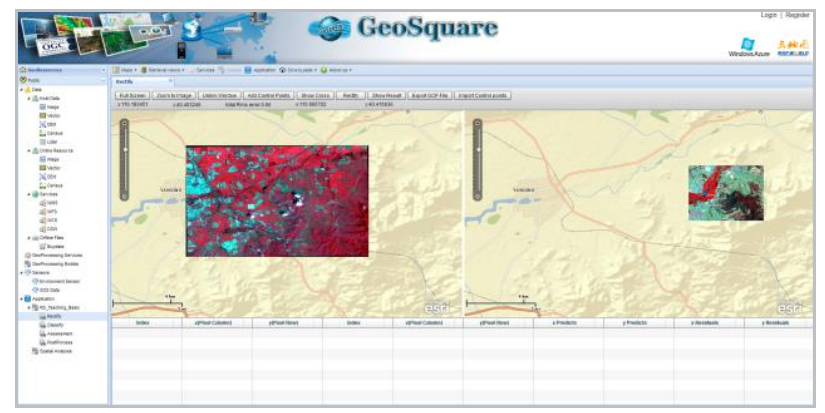

Figure 8 Rectify application of remote sensing image

\subsubsection{Registeration of resource}

The registration center provides register service for image, WMS and atomic service. Data and service providers are allowed to quickly share their data and services through registration.

\section{APPLICATIONS}

GeoSquare has been applied in GIS-related curriculumins at wuhan university successfully. In the ISPRS summer school in May 2014 at Wuhan University, GeoSquare also was used in the teaching and training of basic geographic concepts and operations. We believe that GeoSquare will play an important role in the GIS education and have great potential in assistance of GIS-related research.

\section{ACKNOWLEDGEMENTS}

This research is supported by National Natural Science Foundation of China (41371372).

\section{REFERENCES}

Gong, J. and Wu, H., 2012. The Geospatial Service Web: Ubiquitous Connectivity with Geospatial Services. Transactions in GIS, 16(6), pp. 741--743.

Ivánová, I., Morales, J., de By, R.A., Beshe, T.S. and Gebresilassie, M.A., 2013. Searching for spatial data resources by fitness for use. Journal of Spatial Science, 58(1), pp. 15-28.

Smits, P.C. and Friis-Christensen, A., 2007. Resource Discovery in a European Spatial Data Infrastructure. Knowledge and Data Engineering, IEEE Transactions on, 19(1), pp. 85-95.

Wu, H., Li, Z., Zhang, H., Yang, C. and Shen, S., 2011. Monitoring and evaluating the quality of Web Map Service resources for optimizing map composition over the internet to support decision making. Computers \& Geosciences, 37(4), pp. $485-494$. 